Geopolítica(s) Revista de estudios sobre espacio y poder

ISSN: 2172-3958

\title{
Unidos o Dominados (Discurso pronunciado el 11 de noviembre de 1953 en la Escuela Nacional de Guerra) ${ }^{1}$
}

\author{
Juan Domingo Perón
}

Resumen. Las organizaciones humanas, a lo largo de todos los tiempos, han ido, indudablemente, creando sucesivos agrupamientos y reagrupamientos. El problema fundamental del futuro es un problema de base y fundamento económicos, y la lucha del futuro será cada vez más económica, en razón de una mayor superpoblación y de una mayor superindustrialización. Es indudable que nuestro continente, en especial Sudamérica, es la zona del mundo donde todavía, en razón de su falta de población y de su falta de explotación extractiva, está la mayor reserva de materia prima y alimentos del mundo. Esto nos indicaría que el porvenir es nuestro y que en la futura lucha nosotros marchamos con una extraordinaria ventaja. Pero precisamente en estas circunstancias radica nuestro mayor peligro, porque nosotros estamos amenazados a que un día los países superpoblados y superindustrializados, que tienen un extraordinario poder jueguen ese poder para despojarnos de los elementos de que nosotros disponemos. Es esa circunstancia la que ha inducido a nuestro gobierno a encarar de frente la posibilidad de una unión continental real y efectiva, empezando por Chile, Brasil y Argentina, para encarar una vida en común y para planear, también, una defensa en común.

Palabras clave: Juan Domingo Perón; América Latina; Argentina; política internacional; integración regional.

\section{[en] United or Dominated (Speech delivered on November 11, 1953 at the National War College)}

\begin{abstract}
Human organizations, throughout all time, have undoubtedly been creating successive groupings and regrouping. The fundamental problem of the future is a problem of economic base and foundation, and the struggle of the future will become more and more economical, due to a greater overpopulation and a greater superindustrialization. There is no doubt that our continent, especially South America, is the area of the world where, due to its lack of population and its lack of extractive exploitation, the world's largest reserve of raw materials and food is still. This would indicate to us

1 (Nota de la redacción) El texto original fue publicado bajo el título "Discurso pronunciado por el Excelentísimo Señor Presidente de la Nación General Juan Perón en la Escuela Nacional de Guerra”, por el Ministerio de Defensa Nacional de Argentina en 1953, como Documento Reservado. Hasta 1967, año en que fue dado a conocer públicamente, fue mantenido en secreto. En el folleto original, el texto aquí transcripto venía precedido de la siguiente introducción: “Invitado por el señor Ministro de Defensa Nacional, General de División D. Humberto Sosa Molina, a escuchar una conferencia que dictaría a los cursantes el señor Director de la Escuela Nacional de Guerra, General de División D. Horacio A. Aguirre, el Excelentísimo señor Presidente de la Nación, General de Ejército D. JUAN PERÓN, asistió el 11 de noviembre de 1953 al mencionado Instituto Superior, en compañía del señor Ministro invitante. Terminada la conferencia del señor General Aguirre, el primer magistrado hizo uso de la palabra y vertió los conceptos que se transcriben en este folleto".
\end{abstract}


that the future is ours and that in the future struggle we are marching with an extraordinary advantage. But it is precisely in these circumstances that our greatest danger lies, because we are threatened that one day the overpopulated and superindustrialized countries, which have extraordinary power, will play that power to strip us of the elements that we have. It is this circumstance that has led our government to face head on the possibility of a real and effective continental union, starting with Chile, Brazil and Argentina, to face a life in common and to plan, also, a defense in common.

Keywords: Juan Domingo Perón; Latin America; Argentina; international policy; regional integration.

\section{[pt] Unidos ou Dominados (Discurso proferido em 11 de novembro de 1953 na Escola Nacional de Guerra)}

Resumo. Organizações humanas, durante todo o tempo, sem dúvida criaram agrupamentos e reagrupamentos sucessivos. O problema fundamental do futuro é um problema de base e fundamento econômico, e a luta do futuro se tornará cada vez mais econômica, devido a uma superpopulação maior e uma maior superindustrialização. Não há dúvida de que nosso continente, especialmente a América do Sul, é a região do mundo onde, devido à sua falta de população e à sua exploração extrativa, ainda é a maior reserva mundial de matérias-primas e alimentos. Isso nos indica que o futuro é nosso e que, na luta futura, estamos marchando com uma vantagem extraordinária. Mas é justamente nessas circunstâncias que reside nosso maior perigo, porque somos ameaçados que um dia os países superpovoados e superindustrializados, que têm poder extraordinário, desempenharão esse poder para nos despojar dos elementos que temos. É essa circunstância que levou nosso governo a enfrentar a possibilidade de uma união continental real e efetiva, começando pelo Chile, Brasil e Argentina, enfrentar uma vida em comum e planejar, também, uma defesa em comum.

Palavras-chave: Juan Domingo Perón; América Latina; Argentina; política internacional; integração regional.

Cómo citar: Perón, J. D. (2020). Unidos o Dominados (Discurso pronunciado el 11 de noviembre de 1953 en la Escuela Nacional de Guerra). Geopolítica(s). Revista de estudios sobre espacio y poder, 11(1), 173-183.

\section{Señores:}

He aceptado con gran placer esta ocasión para disertar sobre las ideas fundamentales que han inspirado una nueva política internacional en la República Argentina.

Es indudable que, por el cúmulo de tareas que yo tengo, no podré presentar a ustedes una exposición académica sobre este tema, pero sí podré mantener una conversación en la que lo más fundamental y lo más decisivo de nuestras concepciones será expuesto con sencillez y con claridad.

Las organizaciones humanas, a lo largo de todos los tiempos, han ido, indudablemente, creando sucesivos agrupamientos y reagrupamientos. Desde la familia troglodita hasta nuestros tiempos eso ha marcado un sinnúmero de agrupaciones a través de las familias, las tribus, las ciudades, las naciones y los grupos de naciones, y hay quien se aventura ya a decir que para el año 2000 las agrupaciones menores serán los continentes.

Es indudable que la evolución histórica de la humanidad va afirmando este concepto cada día con mayores visos de realidad. Eso es todo cuanto podemos decir en lo que se refiere a la natural y fatal evolución de la humanidad. Si ese problema lo 
transportamos a nuestra América surge inmediatamente una apreciación impuesta por nuestras propias circunstancias y nuestra propia situación.

Es indudable que el mundo, superpoblado y súper industrializado, presenta para el futuro un panorama que la humanidad todavía no ha conocido por lo menos en una escala tan extraordinaria. Todos los problemas que hoy se ventilan en el mundo son, en su mayoría, producto de esta superpoblación y superindustrialización, sean problemas de carácter material o sean problemas de carácter espiritual. Es tal la influencia de la superproducción y es de tal magnitud la influencia de la técnica y de esa superproducción, que la humanidad, en todos sus problemas económicos, políticos y sociológicos, se encuentra profundamente influida por esas circunstancias.

Si ése es el futuro de la humanidad, es indudable que estos problemas irán progresando y produciendo nuevos y más difíciles problemas emergentes de las circunstancias enunciadas.

Resulta también indiscutible que la lucha fundamental en un mundo superpoblado es por una cosa siempre primordial para la humanidad: la comida. Ese es el peor y el más difícil problema a resolver.

El segundo problema que plantea la industrialización es la materia prima: valdría decir que en este mundo que lucha por la comida y por la materia prima, el problema fundamental del futuro es un problema de base y fundamento económicos, y la lucha del futuro será cada vez más económica, en razón de una mayor superpoblación y de una mayor superindustrialización. En consecuencia, analizando nuestros problemas, podríamos decir que el futuro del mundo, el futuro de los pueblos y el futuro de las naciones estará extraordinariamente influido por la magnitud de las reservas que posean: reservas de alimentos y reservas de materias primas.

Esto es una cosa tan evidente, tan natural y simple, que no necesitaríamos hacer uso ni de la estadística y menos aún de la dialéctica para convencer a nadie.

Y ahora, viendo el problema práctica y objetivamente, pensamos cuáles son las zonas del mundo donde todavía existen las mayores reservas de estos dos elementos fundamentales de la vida humana: el alimento y la materia prima.

Es indudable que nuestro continente, en especial Sudamérica, es la zona del mundo donde todavía, en razón de su falta de población y de su falta de explotación extractiva, está la mayor reserva de materia prima y alimentos del mundo. Esto nos indicaría que el porvenir es nuestro y que en la futura lucha nosotros marchamos con una extraordinaria ventaja a las demás zonas del mundo, que han agotado sus posibilidades de producción alimenticia y de provisión de materias primas o que son ineptas para la producción de estos dos elementos fundamentales de la vida.

Si esto, señores, crea realmente el problema de la lucha, es indudable que en esa lucha llevamos nosotros una ventaja inicial, y que en el aseguramiento de un futuro promisorio tenemos halagüeñas esperanzas de disfrutarlo en mayor medida que otros países del mundo.

Pero precisamente en estas circunstancias radica nuestro mayor peligro, porque es indudable que la humanidad ha demostrado a lo largo de la historia de todos los tiempos que cuando se ha carecido de alimentos o de elementos indispensables para la vida, como serían las materias primas y otros, se ha dispuesto de ellos qui- 
tándolos por las buenas o por las malas, vale decir, con habilidosas combinaciones o mediante la fuerza. Lo que quiere decir, en buen romance, que nosotros estamos amenazados a que un día los países superpoblados y súper industrializados, que no disponen de alimentos ni de materia prima, pero que tienen un extraordinario poder jueguen ese poder para despojarnos de los elementos de que nosotros disponemos en demasía con relación a nuestra población y a nuestras necesidades. Ahí está el problema planteado en sus bases fundamentales, pero también las más objetivas y realistas.

Si subsistiesen los pequeños y débiles países, en un futuro no lejano podríamos ser territorio de conquista como han sido miles y miles de territorios desde los fenicios hasta nuestros días. No sería una historia nueva la que se escribiría en estas latitudes; sería la historia que ha campeado en todos los tiempos, sobre todos los lugares de la tierra, de manera que ni siquiera llamaría mucho la atención.

Es esa circunstancia la que ha inducido a nuestro gobierno a encarar de frente la posibilidad de una unión real y efectiva de nuestros países, para encarar una vida en común y para planear, también, una defensa futura en común.

Si esas circunstancias no son suficientes, o ese hecho no es un factor que gravite decisivamente para nuestra unión, no creo que exista ninguna otra circunstancia importante para que la realicemos.

Si cuanto he dicho no fuese real, o no fuese cierto, la unión de esta zona del mundo no tendría razón de ser, como no fuera una cuestión más o menos abstracta o idealista.

Señores: es indudable que desde el primer momento nosotros pensamos en esto, analizamos las circunstancias y observamos que, desde 1810 hasta nuestros días, nunca han faltado distintos intentos para agrupar esta zona del Continente en una unión de distintos tipos.

Los primeros surgieron en Chile, ya en los días iniciales de las revoluciones emancipadoras de la Argentina, de Chile, del Perú. Todos ellos fracasaron por distintas circunstancias. Es indudable que, de realizarse aquello en ese tiempo, hubiese sido una cosa extraordinaria. Desgraciadamente, no todos entendieron el problema, y cuando Chile propuso eso aquí a Buenos Aires en los primeros días de la Revolución de Mayo, Mariano Moreno fue el que se opuso a toda unión con Chile. Es decir, que estaba en el gobierno mismo, y en la gente más prominente del gobierno, la idea de hacer fracasar esa unión. Eso fracasó por culpa de la Junta de Buenos Aires.

Hubo varios después que fracasaron también por diversas circunstancias. Pasó después el problema a ser propugnado desde Perú, y la acción de San Martín también fracasó. Después fue Bolívar quien se hizo cargo de la lucha por una unidad continental, y sabemos también cómo fracasó.

Se realizaron después el primero, el segundo y el tercer congreso de México con la misma finalidad. Y debemos confesar que todo eso fracasó, mucho por culpa nuestra. Nosotros fuimos los que siempre más o menos nos mantuvimos un poco alejados, con un criterio un tanto aislacionista y egoísta.

Llegamos a nuestros tiempos. Yo no querría pasar a la historia sin haber demostrado, por lo menos fehacientemente, que ponemos toda nuestra voluntad real, efectiva, leal y sincera para que esta unión pueda realizarse en el Continente. 
Pienso yo que el año 2000 nos va a sorprender o unidos o dominados; pienso también que es de gente inteligente no esperar que el año 2000 llegue a nosotros, sino hacer un poquito de esfuerzo para llegar un poco antes del año 2000, y llegar un poco en mejores condiciones que aquella que nos podrá deparar el destino o mientras nosotros seamos yunque que aguantamos los golpes y no seamos alguna vez martillo; que también demos algún golpe por nuestra cuenta.

Es por esa razón que ya en 1946, al hacer las primeras apreciaciones de carácter estratégico y político internacional, comenzamos a pensar en ese grave problema de nuestro tiempo. Quizá en la política internacional que nos interesa, es el más grave y el más trascendente; más trascendente quizá que lo que pueda ocurrir en la guerra mundial, que lo que pueda ocurrir en Europa, o lo que pueda ocurrir en el Asia o en el Extremo Oriente; porque éste es un problema nuestro, y los otros son problemas del mundo en el cual vivimos, pero que están suficientemente alejados de nosotros.

Creo también que en la solución de este grave y trascendente problema cuentan los pueblos más que los hombres y que los gobiernos.

Es por eso que, cuando hicimos las primeras apreciaciones, analizamos si esto podría realizarse a través de las cancillerías actuantes como en el siglo XVIII, en una buena comida, con lucidos discursos, pero que terminan al terminar la comida, inoperantes e intrascendentes, como han sido todas las acciones de las cancillerías de esta parte del mundo desde hace casi un siglo hasta nuestros días; o si habría que actuar más efectivamente, influyendo no a los gobiernos, que aquí se cambian como se cambian las camisas, sino influyendo a los pueblos, que son los permanentes. Porque los hombres pasan y los gobiernos se suceden, pero los pueblos quedan.

Hemos observado, por otra parte, que el éxito, quizás el único éxito extraordinario del comunismo, consiste en que ellos no trabajan con los gobiernos, sino con los pueblos, porque ellos están encaminados a una obra permanente y no a una obra circunstancial.

Y si en el orden internacional quiere realizarse algo trascendente, hay que darle carácter permanente, porque mientras sea circunstancial, en el orden de la política internacional no tendrá ninguna importancia. Por esa razón, y aprovechando las naturales inclinaciones de nuestra doctrina propia, comenzamos a trabajar sobre los pueblos, sin excitación, sin apresuramientos y, sobre todo, tratando de cuidar minuciosamente, de desvirtuar toda posibilidad de que nos acusen de intervención en los asuntos internos de otros Estados.

En 1946 cuando yo me hice cargo del gobierno, la política internacional argentina no tenía ninguna definición.

No encontramos allí ningún plan de acción, cómo no existía tampoco en los ministerios militares ni siquiera una remota hipótesis sobre la cual los militares pudieran basar sus planes de operaciones. Tampoco en el Ministerio de Relaciones Exteriores, en todo su archivo, había un solo plan activo sobre la política internacional que seguía la República Argentina, ni siquiera sobre la orientación, por lo menos, que regían sus decisiones o designios.

Vale decir que nosotros habíamos vivido, en política internacional, respondiendo a las medidas que tomaban los otros con referencia a nosotros, pero sin tener jamás una idea propia que nos pudiese conducir, por lo menos a lo largo de los tiempos, con una dirección uniforme y congruente. Nos dedicamos a tapar los agu- 
jeros que nos hacían las distintas medidas que tomasen los demás países. Nosotros no teníamos iniciativa.

No es tan criticable el procedimiento, porque también suele ser una forma de proceder, quizás explicable, pues los pequeños países no pueden tener en el orden de la política internacional objetivos muy activos ni muy grandes; pero tienen que tener algún objetivo.

Yo no digo que nos vamos a poner nosotros a establecer objetivos extracontinentales para imponer nuestra voluntad a los rusos, a los ingleses o a los norteamericanos; no, porque eso sería torpe.

Vale decir que en esto, como se ha dicho y sostenido tantas veces, hay que tener la política de la fuerza que se posee o la fuerza que se necesita para sustentar una política.

Nosotros no podemos tener lo segundo y, en consecuencia, tenemos que reducirnos a aceptar lo primero, pero dentro de esa situación podemos tener nuestras ideas y luchar por ellas para que las cancillerías, que juegan al estilo del siglo XVIII, no nos estén dominando con sus sueños fantásticos de hegemonía, de mando y de dirección.

Para ser país monitor - como sucede con todos los monitores - ha de ser necesario ponerse adelante para que los demás lo sigan. El problema es llegar cuanto antes a ganar la posición o la colocación y los demás van a seguir aunque no quieran. De manera que la hegemonía no se discute: la hegemonía se conquista o no se conquista. Por eso nuestra lucha no es, en el orden de la política internacional, por la hegemonía de nadie, como lo he dicho muchas veces, sino simplemente y llanamente la obtención de lo que conviene al país en primer término; en segundo término, lo que conviene a la gran región que encuadra el país y, en tercer término, el resto del mundo, que ya está más lejano y a menor alcance de nuestras previsiones y de nuestras concepciones.

Por eso, bien claramente entendido, como lo he hecho en toda circunstancia, para nosotros, primero la República Argentina, luego el Continente y después el mundo. En esa posición nos han encontrado y nos encontrarán siempre, porque entendemos que la defensa propia está en nuestras manos; que la defensa, diremos relativa, está en la Zona Continental que defendemos y en que vivimos, y que la absoluta es un sueño que todavía no ha alcanzado ningún hombre ni nación alguna de la tierra. Vivimos solamente en una seguridad relativa, pensando, señores, en la idea fundamental de llegar a una unión en esta parte del Continente.

Habíamos pensado que la lucha del futuro será económica; la historia nos demuestra que ningún país se ha impuesto en ese campo, ni en ninguna lucha, si no tiene en sí una completa, diremos, unidad económica.

Los grandes imperios, las grandes naciones, han llegado desde los comienzos de la historia hasta nuestros días, a las grandes conquistas, sobre la base de una unidad económica. Y yo analizo que si nosotros soñamos con la grandeza - que tenemos obligación de soñar- para nuestro país, debemos analizar primordialmente ese factor en una etapa del mundo en que la economía pasará a primer plano en todas las luchas del futuro.

La República Argentina sola, no tiene unidad económica; Brasil solo, no tiene tampoco unidad económica; Chile solo, tampoco tiene unidad económica; pero estos tres países unidos conforman quizá en el momento actual la unidad económi- 
ca más extraordinaria del mundo entero, sobre todo para el futuro, porque toda esa inmensa disponibilidad constituye su reserva. Estos son países reserva del mundo.

Los otros están quizá a no muchos años de la terminación de todos sus recursos energéticos y de materia prima; nosotros poseemos todas las reservas de las cuales todavía no hemos explotado nada.

Esa explotación que han hecho de nosotros, manteniéndonos para consumir lo elaborado por ellos, ahora en el futuro puede dárseles vuelta, porque en la humanidad y en el mundo hay una justicia que está por sobre todas las demás justicias, y que algún día llega. Y esa justicia se aproxima para nosotros; solamente debemos tener la prudencia y la sabiduría suficientes para prepararnos a que no nos birlen de nuevo la justicia, en el momento mismo en que estamos por percibirla y por disfrutarla.

Esto es lo que ordena, imprescriptiblemente, la necesidad de la unión de Chile, Brasil y Argentina.

Es indudable que, realizada esta unión, caerán en su órbita los demás países sudamericanos, que no serán favorecidos ni por la formación de un nuevo agrupamiento y probablemente no lo podrán realizar en manera alguna, separados o juntos, sino en pequeñas unidades.

Apreciado esto, señores, yo empecé a trabajar sobre los pueblos. Tampoco olvidé de trabajar a los gobiernos, y durante los seis años del primer gobierno, mientras trabajábamos activamente en los pueblos, preparando la opinión para bien recibir esta acción, conversé con los que iban a ser presidentes, por lo menos, en los dos países que más nos interesaban: Getulio Vargas y el General Ibáñez.

Getulio Vargas estuvo total y absolutamente de acuerdo con esta idea y en realizarla tan pronto él estuviera en el gobierno; Ibáñez me hizo exactamente igual manifestación, y contrajo el compromiso de proceder lo mismo.

Yo no me hacía ilusiones porque ellos hubieran prometido esto para dar el hecho por cumplido porque bien sabía que eran hombres que iban al gobierno y no iban a poder hacer lo que quisieran, sino lo que pudieran. Sabía bien que un gran sector de esos pueblos se iba a oponer tenazmente a una realización de este tipo, por cuestiones de intereses personales y negocios, más que por ninguna otra causa. ¡Cómo no se van a oponer los ganaderos chilenos a que nosotros exportemos sin medida ganado argentino a Chile! ¡Y cómo no se van a oponer a que solucionemos todos los problemas fronterizos para la internación de ganado, los acopiadores chilenos, cuando una vaca o un novillo, a un metro de la frontera chilena hacia el lado argentino, vale diez mil pesos chilenos, y a un metro hacia Chile de la frontera argentina, vale veinte mil pesos chilenos! Ese que gana los diez mil pesos no va a estar de acuerdo nunca con una unidad de este tipo.

Cito este caso grosero para que los señores intuyan toda la gama inmensa de intereses de todo orden que se desgranan en cada una de las cosas que come el pobre "roto" chileno y que producimos nosotros, o que consumimos nosotros y producen ellos. Ese mismo fenómeno sucede con el Brasil.

Por esta razón nunca me hice demasiadas ilusiones sobre las posibilidades de ello; por eso seguimos trabajando por estas uniones, porque ellas deberán venir por los pueblos. 
Nosotros tenemos muy triste experiencia de las uniones que han venido por los gobiernos; por lo menos, ninguna en ciento cincuenta años ha podido cristalizar en alguna realidad.

Probemos el otro camino que nunca se ha probado. Para ver si, desde abajo, podemos ir influyendo en forma determinante para que esas uniones se realicen.

Señores: sé también que el Brasil, por ejemplo, tropieza con una gran dificultad. Es ltamaraty, que allí constituye una institución supergubernamental. 1tamaraty ha soñado, desde la época de su Emperador hasta nuestros días, con una política que se ha prolongado a través de todos los hombres que han ocupado ese difícil cargo en el Brasil.

Ella los había llevado a establecer un arco entre Chile y el Brasil; esa política debe ser vencida con el tiempo y por un buen proceder de parte nuestra.

Debe desmontarse todo el sistema de ltamaraty y deben desaparecer esas excrecencias imperiales que constituyen, más que ninguna otra razón, los principales obstáculos para que Brasil entre a una, diremos, unión verdadera con la Argentina.

Nosotros con ellos no tenemos ningún problema, como no sea ese sueño de la hegemonía, en el que estamos prontos a decirles: son ustedes más grandes, más lindos y mejores que nosotros, no tenemos ningún inconveniente.

Nosotros renunciamos a todo eso, de manera que ese tampoco va a ser un inconveniente. Pero es indudable que nosotros creíamos superado en cierta manera ese problema.

Yo he de contarles a los señores un hecho que pondrá perfectamente en evidencia cómo procedemos nosotros y por qué tenemos la firme convicción de que al final vamos a ganar nosotros, porque procedemos bien. Porque los que proceden mal son los que sucumben víctimas de su propio mal procedimiento: por eso, no emplearemos en ningún caso ni los subterfugios, ni las insidias, ni las combinaciones raras, que emplean algunas cancillerías.

Cuando Vargas subió al gobierno me prometió a mí que nos reuniríamos en Buenos Aires, o en Río, y haríamos ese tratado que yo firmé con Ibáñez después: el mismo tratado.

Ese fue un propósito formal que nos habíamos trazado. Más aún, dijimos: "Vamos a suprimir las fronteras, si es preciso". Yo "agarraba" cualquier cosa, porque estaba dentro de la orientación que yo seguía y de lo que yo creía que era necesario y conveniente.

Yo sabía que acá yo lo realizaba, porque cuando le dijera a mi pueblo que quería hacer eso, sabía que mi pueblo querría lo que yo quería en el orden de la política internacional, porque ya aquí existe una conciencia político-internacional en el pueblo, y existe una organización. Además, la gente sabe que, en fin, tantos errores no cometemos, de manera que tiene también un poco de fe en lo que hacemos.

Más tarde Vargas me dijo que era difícil que pudiéramos hacerlo tan pronto, porque él tenía una situación política un poco complicada en las Cámaras y que antes de dominarlas quería hacer una conciliación. Es difícil eso en política; primero hay que dominar y después la conciliación viene sola. Son puntos de vista; son distintas maneras de pensar.

Él siguió un camino distinto y nombró un gabinete de conciliación, vale decir, nombró un gabinete donde por lo menos las tres cuartas partes de los ministros eran 
enemigos políticos de él, y que servirían a sus propios intereses y no a los del gobierno.

Claro que él creyó que esto en seis meses le iba a dar la solución. Pero cuando pasaron los seis meses el asunto estaba más complicado que antes. Naturalmente, no pudo venir acá; no pudo comprometerse frente a su Parlamento ni frente a sus propios ministros a realizar una tarea que implicaba ponerse los pantalones y jugarse una carta decisiva frente a la política internacional mundial, a su pueblo, a su Parlamento y a los intereses que había que vencer.

Naturalmente, yo esperé. En ese ínterin es elegido presidente el general Ibáñez; la situación de él no era mejor que la situación de Vargas, pero en cierta manera llegaba plebiscitado en todo lo que se puede ser plebiscitado en Chile, con elecciones muy sui géneris, porque allá se inscriben los que quieren, y los que no quieren no; es una cosa muy distinta a la nuestra. Pero él llega al gobierno naturalmente. Tan pronto llega al gobierno, yo, conforme con lo que habíamos conversado, lo tanteé. Me dijo: "De acuerdo; lo hacemos. ¡Muy bien!” El General fue más decidido, porque los generales solemos ser más decididos que los políticos. Pero antes de hacerlo, como tenía un compromiso con Vargas, le escribí una carta que le hice llegar por intermedio de su propio embajador, a quien llamé y dije: "Vea, usted tendrá que ir a Río con esta carta y tendrá que explicarle todo esto a su presidente. Hace dos años nosotros nos prometimos realizar este acto. Hace más de un año y pico que lo estoy esperando, y no puede venir. Yo le pido autorización a él para que me libere de ese compromiso de hacerlo primero con el Brasil, y me permita hacerlo primero con Chile. Claro que le pido esto porque creo que estos tres países son los que deben realizar la unión"

El embajador va allá y vuelve y me dice, en nombre de su presidente, que no solamente me autoriza a que vaya a Chile, liberándome del compromiso, sino que me da también su representación para que lo haga en nombre de él, en Chile. Naturalmente, ya sé ahora muchas cosas que antes no sabía; acepté sólo la autorización, pero no la representación.

Fui a Chile, llegué allí, y le dije al general Ibáñez: "Vengo aquí con todo listo y traigo la autorización del presidente Vargas, porque yo estaba comprometido a hacer esto primero con él y con el Brasil; de manera que todo sale perfectamente bien y como lo hemos planeado, y quizá al hacerse esto se facilite la acción de Vargas y se vaya arreglando así mejor el asunto".

Llegamos, hicimos allá con el ministro de Relaciones Exteriores todas esas cosas de las cancillerías, discutimos un poco — poca cosa - y llegamos al acuerdo, no tan amplio como nosotros queríamos, porque la gente tiene miedo en algunas cosas y, es claro, salió un poco retaceado, pero salió. No fue tampoco un parto de los montes, pero costó bastante convencer, persuadir, etc.

Y al día siguiente llegan las noticias de Río de Janeiro, donde el ministro de Relaciones Exteriores del Brasil hacía unas declaraciones tremendas contra el Pacto de Santiago: que estaba en contra de los pactos regionales, que ése era la destrucción de la unanimidad panamericana. Imagínense la cara que tendría yo al día siguiente cuando fui y me presenté al presidente Ibáñez. Al darle los buenos días, me preguntó: "¿Qué me dice de los amigos brasileños?" 
Naturalmente que la prensa carioca sobrepasó los límites a que había llegado el propio ministro de Relaciones Exteriores, señor Neves de Fontoura. Claro, yo me callé; no tenía más remedio. Firmé el tratado y me vine aquí.

Cuando llegué me encontré con Gerardo Rocha, viejo periodista de gran talento, director de $O$ Mundo en Río, muy amigo del presidente Vargas, quien me dijo: "Me manda el presidente Vargas para que le explique lo que ha pasado en el Brasil. Dice que la situación de él es muy difícil: que políticamente no puede dominar, que tiene sequías en el norte, heladas en el sur; y a los políticos los tiene levantados; que el comunismo está muy peligroso, que no ha podido hacer nada; en fin, que lo disculpe, que él no piensa así, y que si el ministro ha hecho eso, que él tampoco puede mandar al ministro".

Yo me he explicado perfectamente bien todo esto; no lo justificaba, pero me lo explicaba por lo menos. Naturalmente, señores, que planteada la situación en estas circunstancias, de una manera tan plañidera y lamentable, no tuve más remedio que decirle que siguiera tranquilo, que yo no me meto en las cosas de él y que hiciera lo que pudiese, pero que siguiera trabajando por esto.

Bien, señores, yo quería contarles esto, que probablemente no lo conoce nadie más que los ministros y yo. Claro está que son todos documentos para la historia, porque yo no quiero pasar a la historia como un cretino que ha podido realizar esta unión y no la ha realizado. Por lo menos quiero que la gente piense en el futuro que si aquí ha habido cretinos, no he sido yo sólo; hay otros cretinos también como yo, $\mathrm{y}$ todos juntos iremos en el baile del cretinismo.

Pero lo que yo no quería es dejar de afirmar, como lo haré públicamente en alguna circunstancia, que toda la política argentina en el orden internacional ha estado orientada hacia la necesidad de esa unión. Para que cuando llegue el momento en que seamos juzgados por nuestros hombres - frente a los peligros que esta disociación producirá en el futuro-, por lo menos tengamos el justificativo de nuestra propia impotencia para realizarla.

Sin embargo, yo no soy pesimista: yo creo que nuestra orientación, nuestra perseverancia, va todos los días ganando terreno dentro de esta idea, y estoy casi convencido de que un día lo hemos de realizar todo bien y acabadamente, y que tenemos que trabajar incansablemente por realizarlo. Ya se acabaron las épocas del mundo en que los conflictos eran entre dos países. Ahora los conflictos se han agrandado de tal manera y han adquirido tal naturaleza que hay que prepararse para los "grandes conflictos" y no para los pequeños conflictos.

Esta unión, señores, está en plena elaboración. Es todo cuanto yo podría decirles a ustedes como definitivo.

Estamos trabajándola, y el éxito, señores, ha de producirse; por lo menos, nosotros hemos preparado el éxito, lo estamos realizando, y no tengan la menor duda de que el día que se produzca yo he de saber explotarlo con todas las conveniencias necesarias para nuestro país. Porque, de acuerdo con el aforismo napoleónico, el que prepara un éxito y lo conquista, difícilmente no sabe sacarle las ventajas cuando lo ha obtenido.

En esto, señores, estoy absolutamente persuadido de que vamos por buen camino. La contestación del Brasil, buscando desviar su arco de Santiago a Lima, es solamente una contestación ofuscada y desesperada de una cancillería que no inter- 
preta el momento, y que está persistiendo sobre una línea superada por el tiempo y por los acontecimientos; eso no puede tener efectividad.

La lucha por las zonas amazónicas y del Plata no tiene ningún valor ni ninguna importancia; son sueños un poco ecuatoriales y nada más. No puede haber en ese sentido ningún factor geopolítico ni de ninguna otra naturaleza que pueda enfrentar a estas dos zonas tan diversas en todos sus factores, y en todas sus características.

Aquí hay un problema de unidad que está por sobre todos los problemas, y en estas circunstancias, quizá muy determinantes, de haber nosotros solucionado nuestros entredichos con Estados Unidos, tal vez esto favorezca en forma decisiva la posibilidad de una unión continental en esta zona del continente americano.

Señores: como ha respondido el Paraguay, aunque es un pequeño país; como irán respondiendo otros países del continente, despacito, sin presiones y sin violencias de ninguna naturaleza, así se va configurando ya una suerte de unión.

Las uniones deben realizarse por el procedimiento que es común: primeramente hay que conectar algo; después las demás conexiones se van formando con el tiempo y con los acontecimientos. tina.

Chile, aun a pesar de la lucha que debe sostener allí, ya está unido con la Argen-

El Paraguay se halla en igual situación. Hay otros países que ya están inclinados a realizar lo mismo. Si nosotros conseguimos ir adhiriendo lentamente a otros países, no va a tardar mucho en que el Brasil haga también lo mismo, y ése será el principio del triunfo de nuestra política.

La unión continental sobre la base de la Argentina, Brasil y Chile está mucho más próxima de lo que creen muchos argentinos, muchos chilenos y muchos brasileños; en el Brasil hay un sector enorme que trabajó por esto.

Lo único que hay que vencer son intereses; pero cuando los intereses de los países entran a actuar, los de los hombres deben ser vencidos por aquellos; ésa es nuestra mayor esperanza.

Hasta que esto se produzca, señores, no tenemos otro remedio que esperar y trabajar para que se realice: esa es nuestra acción y esa es nuestra orientación.

Muchas gracias. 